\title{
What job can a bug give? A controversy over the arsenic-guzzling bacterium cultured by NASA
}

\author{
Mingwei Sun ${ }^{1,2}$, Christopher J. Vavricka ${ }^{1}$, Baoli Zhu ${ }^{1 凶}$ \\ ${ }^{1}$ CAS Key Laboratory of Pathogenic Microbiology and Immunology, Institute of Microbiology, Chinese Academy of Sciences \\ (CASPMI), Beijing 100101, China \\ ${ }^{2}$ College of Life Sciences, University of Science and Technology of China, Hefei 230027, China \\ Correspondence: zhubaoli@im.ac.cn
}

The mysteries of life's evolution on our planet drive us to explore the limits of biology. Life forms on Earth as we know are composed of six essential elements: carbon, hydrogen, oxygen, nitrogen, sulfur and phosphorus. However, a recently published research work funded by the National Aeronautics and Space Administration (NASA), USA, has almost shaken the fundamental knowledge about what is life made of on Earth. The research team led by NASA astrobiologist Felisa Wolfe-Simon described a microbe isolated from California's Mono Lake that could sustain its growth by using toxic chemical arsenic (As) instead of phosphorus $(P)$. This surprising discovery was published online by Science Express on December 2 last year (Wolfe-Simon et al., 2010 , online). If the authors of the paper were right, we would have to expand our definition of life on this planet. The arsenic-bug they grew was dubbed GFAJ-1, which interestingly stands for "Give Felisa A Job."

Felisa Wolfe-Simon, an American biogeochemist and microbial geobiologist, is a member of the NASA Astrobiology Institute whose research focuses on evolutionary microbiology and exotic metabolic pathways. During the last two or three years, Wolfe-Simon and colleagues had proposed that arsenate $\left(\mathrm{AsO}_{4}^{3-}\right)$ could be able to substitute for phosphate $\left(\mathrm{PO}_{4}^{3-}\right)$ in various biochemical pathways. As belongs to the same group as $\mathrm{P}$ on the periodic table and shares some similar chemical properties with P. Located in California, United States, the arsenic-rich Mono Lake has a bizarre and extraterrestrial beauty that captured Wolfe-Simon's interests, not only because of its scenery, but for the potential presence of shadowy biosphere containing exotic life forms. The researchers led by Wolfe-Simon used Mono Lake bottom sediments as inocula in an aerobic artificial medium containing limited nutrition and a gradient of added $\mathrm{AsO}_{4}^{3-}$ but without $\mathrm{PO}_{4}^{3-}$. After several dilution transfers of an original culture, GFAJ-1 was isolated as one of the fastest growing single colonies for further tests. The phylogenetic analysis based on 16S rRNA sequences shows that GFAJ-1 is closely related to other halophile bacteria of the Halomonadaceae family in Gammaproteobacteria rather than a novel species. Actually, GFAJ-1 presents an unprecedented capacity to survive in the presence of high levels of As while starved of $\mathrm{P}$ compared to the other Halomonadaceae family members according to the Science journal article. To demonstrate this, the team designed three experimental conditions: added $\mathrm{AsO}_{4}^{3-}$ without $\mathrm{PO}_{4}^{3-}$ (+As/-P); added $\mathrm{PO}_{4}^{3-}$ without $\mathrm{AsO}_{4}^{3-}(-\mathrm{As} /+\mathrm{P})$, as test groups; and added neither $\mathrm{AsO}_{4}^{3-}$ nor $\mathrm{PO}_{4}^{3-}(-\mathrm{As} /-\mathrm{P})$ as a control group. A growth experiment at $28^{\circ} \mathrm{C}$ indicated that the GFAJ-1 cell volume was 1.5 times greater under $+\mathrm{As} /-\mathrm{P}$ conditions than that of $-\mathrm{As} /+\mathrm{P}$, although they only grew $60 \%$ as fast as when in phosphoric solution. The data showed little increase in cell number or optical density when the bacteria were cultured in the control environment in which neither $\mathrm{PO}_{4}^{3-}$ nor $\mathrm{AsO}_{4}^{3-}$ was added. Furthermore, the cell number and optical density of the control was much lower than that in the $\mathrm{PO}_{4}^{3-}$ and $\mathrm{AsO}_{4}^{3-}$ conditions. Considering these results, the authors speculated that as the trace $P$ in the medium could not maintain growth and that GFAJ-1 in +As/-P medium might take As up into their cells. Further analyses by ICP-MS (inductively coupled plasma mass spectrometry) and highintensity synchrotron X-ray experiments suggested there was high As content with low background P. Moreover, the researchers further tracked the As distribution by adding isotope-labeled ${ }^{73} \mathrm{AsO}_{4}^{3-}$ and found that $\mathrm{As}$ was present in 
many macromolecules as well as small molecular metabolites such as ATP, NADH, glucose and acetyl-CoA, which normally contain P. Even more striking, it was found that, according to the authors, GFAJ-1 grown in + As/-P conditions could use As as a replacement for $P$ to form the backbone of those biomolecules, specifically in DNA, which was suggested by the data based on NanoSIMS (high resolution secondary ion mass spectrometry). In addition, the Micro X-ray absorption data provided in the article allegedly supported the above results (Wolfe-Simon et al., 2010 online). NASA thereupon held a press conference on that very day when their Science paper published online to make this discovery more visible to the public, despite the fact that the mechanisms by which As integrates into the structure of those biomolecules were still not established. The intriguing finding of such an extreme microbe might suggest that the potential life on other planets could have a chemical composition differing from that of known organisms on Earth, as the authors hinted, increasing the probability of finding extraterrestrial life. It has also been speculated that this possible phenomenon provides more specific information about the origin of life occurring on early Earth (Pennisi, 2010).

As soon as the news was widely publicized, it was also promptly responded to with skepticism and even criticism by scientists and journalists. One of the objections was regarding the chemical role that As plays in the bacterium's cells. As is chemically similar to $P$, however it is still toxic. As and many of its compounds are especially potent poisons. For instance, $\mathrm{AsO}_{4}^{3-}$ is a potent uncoupler of oxidative phosphorylation competing with $\mathrm{PO}_{4}^{3-}$, thus disrupting energy-associated ATP synthesis and NADH production (Miller et al., 2002). Furthermore, a similar mechanism could occur in glycolysis or the tricarboxylic acid cycle (Hughes, 2002). Therefore, some chemists have expressed doubts about how GFAJ-1 uses As to build some of its cellular components. Besides this, other critics argued that the $\mathrm{AsO}_{4}^{3-}$ esters are much more unstable than $\mathrm{PO}_{4}^{3-}$ esters in water and hence DNA with an $\mathrm{AsO}_{4}^{3-}$ backbone will hydrolyze quickly. In fact, experiments have demonstrated that the hydrolysis of arsenic-containing nucleotides occurs with a half-life in the tens of minutes range (Lagunas et al., 1984). The NASA researchers immersed the extracted DNA samples in water and the DNA remained stable, which suggests that it contained stable $\mathrm{PO}_{4}^{3-}$ ester bonds. Additionally, some experts questioned whether there was any direct proof in the research data that the As was really a part of the molecular components. They suspected that GFAJ-1 was just an arsenic-resistant bacterium which scavenges trace phosphoric contaminants from its surroundings to survive in $\mathrm{PO}_{4}^{3-}$ starved medium. As a matter of fact, many bacteria live in Mono Lake, in which there is a high $\mathrm{AsO}_{4}^{3-}$ concentration, but many naturally use $\mathrm{P}$ instead of As for making DNA. NASA's Science paper deduced that the intracellular $P$ content of the cells in +As/-P medium was far below the requirement for supporting growth which should be $1 \%-3 \%$ by dry weight as the authors explained. However, the numbers are not even true because the corresponding value in -As/+ P medium was only $0.5 \%$ on average in the same study. Otherwise, the ratio of radiolabeled arsenic to carbon $\left({ }^{75} \mathrm{As}-{ }^{12} \mathrm{C}-\right)$ in + As/-P condition was only twice higher than the $-A s /+P$ counterpart when measured by NanoSIMS, which seems to be an experimental error. Furthermore, DNA analysis indicated that the cells contained $0.19 \%$ As by dry weight, but the DNA only contained $27 \mathrm{ppb}$ (part per billion) As. Therefore, very little of the cellular As was likely incorporated into DNA and possibly was non-covalently complexed with the DNA, while the majority was probably simply sequestered elsewhere in the cells. Further critics of the investigation questioned whether the protocols lacked the proper washing steps during gel purification and the absence of a comparable control experiment using isotope-labeled $\mathrm{PO}_{4}^{3-}$. In summary, nearly all of the comments reflected by the NASA team had failed to provide a convincing explanation to support their conclusions. In response, a FAQ page was published on December 16 by NASA scientists who wanted to improve understanding of their work (http://ironlisa.com/gfaj/GFAJquestions_Response_16Dec2010.pdf) and modified their previous expression into "GFAJ-1 can substitute As for a small percentage of its $P$ and sustain its growth," but maintained that various types of evidence in their study were veracious and reliable, either in DNA extraction steps or in other measurements. Furthermore, they replied to several queries about the hydrolysis of $\mathrm{AsO}_{4}^{3-}$ esters and speculated that the geometrical constraints of arsenate-linked DNA are likely to slow the rates of hydrolysis, or that GFAJ-1 may have unknown biological mechanisms to compensate. Despite all of these, their announcement suggesting evidence for extraterrestrial life was not accepted, but instead was criticized as misleading.

Although none of the scientists ruled out the possibility that such exotic bacteria might exist, the reported study did not provide enough evidence to support the claims made by the authors. Consequently, some straightforward tests on the bacterium are required to answer whether they really do have arsenic-containing DNA once and for all, such as high resolution X-Ray crystal structure of DNA with actual As atoms in the $\mathrm{P}$ positions. Moreover, mass spectra of GFAJ-1's DNA sequences would also confirm unambiguously that nucleotides contain $\mathrm{AsO}_{4}^{3-}$ or $\mathrm{PO}_{4}^{3-}$. NASA scientists had indicated that GFAJ-1 and its DNA samples would be made available to researchers who want to study it. Therefore, other scientists should be motivated to test this finding so that the verdict could be revealed soon. If the results are substantiated, GFAJ-1 might be the only known organism to be capable of incorporating $\mathrm{AsO}_{4}^{3-}$ as a substitute for a small percentage of $\mathrm{PO}_{4}^{3-}$ in its DNA and potentially other biomolecules. However, if not, more insights should be promoted to clarify the facts in this study and to advance 
scientific understanding. And the "job" given by GFAJ-1, a weird life, in any events should not be a "snow job," to either Felisa or the community.

\section{REFERENCES}

Wolfe-Simon, F., Blum, J.S, Kulp, T.R., Gordon, G.W., Hoeft, S.E., Pett-Ridge, J., Stolz, J.F., Webb, S.M., Weber, P.K., Davies, P.C. W., Anbar, A.D., Oremland, R.S. A Bacterium That Can Grow by Using Arsenic Instead of Phosphorus. Science published online 2 December 2010.
Hughes, M.F. (2002). Arsenic toxicity and potential mechanisms of action. Toxicol Lett 133, 1-16.

Lagunas, R., Pestana, D., and Diez-Masa, J.C. (1984). Arsenic mononucleotides. Separation by high-performance liquid chromatography and identification with myokinase and adenylate deaminase. Biochemistry 23, 955-960.

Miller, W.H., Jr., Schipper, H.M., Lee, J.S., Singer, J., and Waxman, S. (2002). Mechanisms of action of Arsenic trioxide. Cancer Res 62, 3893-3903.

Pennisi, E. (2010). What Poison? Bacterium Uses Arsenic to Build DNA and Other Molecules. Science 330, 1302. 